\title{
Imaging Agent
}

National Cancer Institute

\section{Source}

National Cancer Institute. Imaging Agent. NCI Thesaurus. Code C1966.

A compound that enables or improves the visualization of body structures or functions for medical imaging. 